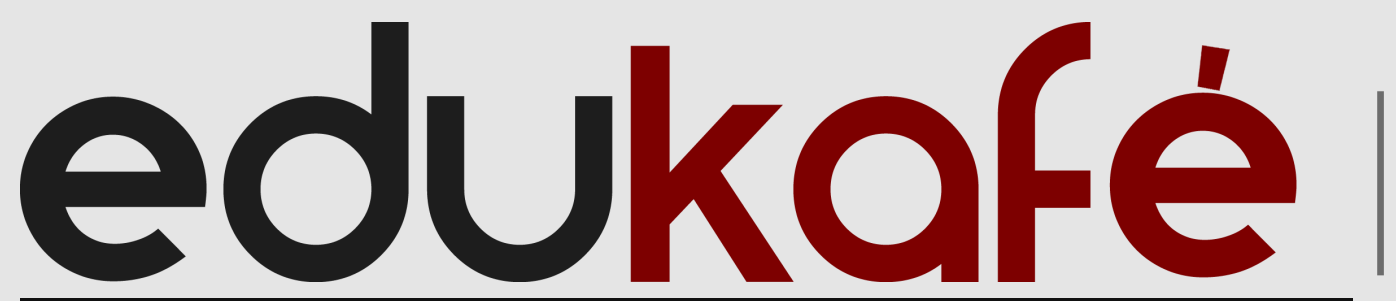

documentos de trabajo de la Escuela

Cali, Colombia

ISSN 2711-2799 (en línea)

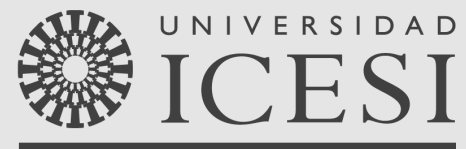

número 1

Febrero 2018

\title{
Impacto de los cursos de comunicación oral y escrita (COE) entre estudiantes de la Universidad Icesi
}

Hoover Delgado Madroñero

Juan Carlos Penagos y Alberto Rodríguez Grupo de investigación IRTA, Universidad Icesi

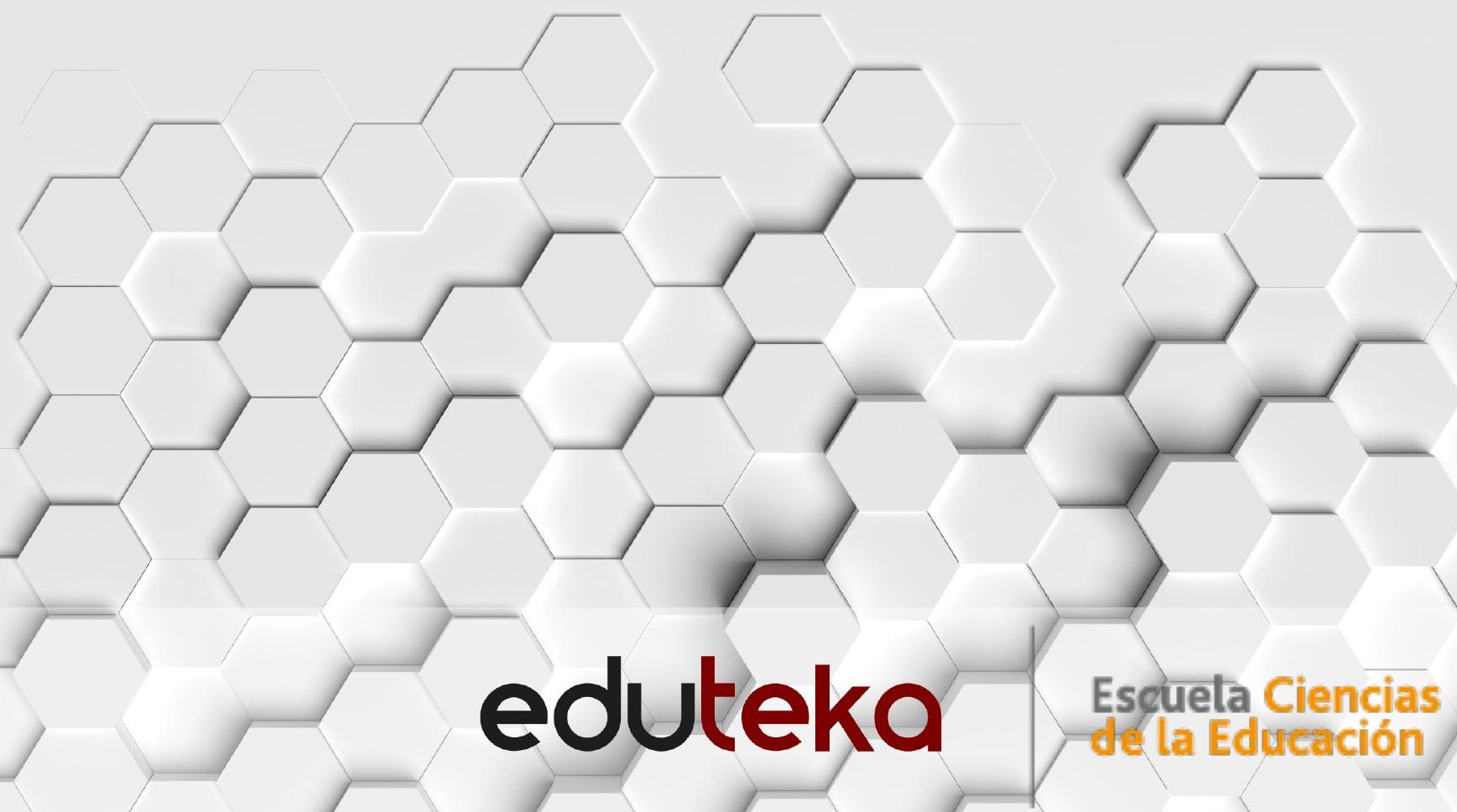


Número 1 • Febrero 2018

ISSN: 2711-2799 (en línea)

DOI: http://doi.org/10.18046/edukafe.2018.1

Rector: Francisco Piedrahita Plata

Secretaria general: María Cristina Navia Klemperer

Director académico: José Hernando Bahamón Lozano

Directora de la Escuela de Ciencias de la Educación: Ana Lucía Paz Rueda

Director del Centro Eduteka: Alejandro Dominguez Z.

Editor del portal Eduteka: Juan Carlos López-García • http://eduteka.icesi.edu.co

\section{Comité Editorial}

Ana Lucía Paz Rueda

Diana Margarita Díaz

Hoover Delgado Madroñero

Jhonny Segura

José Darío Saenz

Edición: Centro Eduteka

Editorial Universidad Icesi • Coordinación editorial: Adolfo A. Abadía

editorial@icesi.edu.co•https://www.icesi.edu.co/editorial

Diseño editorial y diseño portada: Juan Carlos López-García

Diseño del logo edukafé: Boris Sánchez Molano y Carlos Andrés Ávila

Imágenes de portada y contraportada: Magic Creative • Tokyo/Japan • pixabay.com

\section{Universidad Icesi}

Centro Eduteka

Escuela de Ciencias de la Educación (ECE)

Calle 18 No. 122-135 Pance $\bullet$ Cali - Colombia

Teléfono: +57 (2) 5552334 • Fax: +57 (2) 5551441

jclopez@icesi.edu.co•http://eduteka.icesi.edu.co/articulos/edukafe

\author{
Vladimir Rouvinski \\ José Benito Garzón M. (Unicatólica)
}

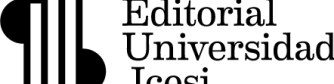
Icesi

Favor citar este documento de trabajo de la siguiente forma:

Delgado Madroñero, Hoover Alfonso; Penagos Trujillo, Juan Carlos y Rodríguez, Alberto (2018). Impacto de los cursos de comunicación oral y escrita entre estudiantes de la Universidad Icesi. Edukafé, Documentos de trabajo de la Escuela, No. 1. Cali: Universidad Icesi. Recuperado, el 25 de Febrero de 2018, de Eduteka: http://doi.org/10.18046/edukafe.2018.1

El Centro Eduteka no se hace responsable de las ideas expuestas, los modelos teóricos presentados o los nombres aludidos por el(los) autor(es) de los artículos publicados en la colección edukafé. El contenido de esta publicación es responsabilidad exclusiva del(Ios) autor (es), y no reflejan la opinión de las directivas de la Universidad Icesi, del Centro Eduteka, de la Escuela de Ciencias de la Educación (ECE), o de los editores de EDUKAFÉ - DOCUMENTOS DE TRABAJO DE LA ESCUELA

Licencia Creative Commons • Atribución/No-comercial/Compartir-igual Esta licencia le permite distribuir, remezclar, retocar, y crear a partir de este este documento de modo no comercial, siempre y cuando se dé el crédito explícito a su(s) autor(xs) y a la Universidad Icesi y se licencien las nuevas creaciones bajo las mismas condiciones.

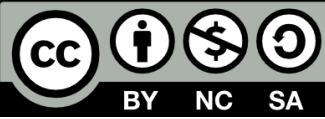




\section{Contenido}

Problema de investigación $\quad 5$

Marco teórico y estado del arte 6

Objetivos 16

Metodología del proyecto 17

Resultados 17

Conclusión 25

Bibliografía 27

\section{edukafé}

"Frente a una taza con café se piensa, pero también se discute, se recuerda o se argumenta. Frente a la taza con café se columbra, se reflexiona, se sueña, se imagina, se escribe, se conversa... Y el café, el misterioso café escucha, profetiza, atestigua, aconseja, da fe, observa, asiente..."

Gustavo Máynez Tenorio

\section{http://eduteka.icesi.edu.co/articulos/edukafe}

Este documento de trabajo hace parte de la colección "edukafe" consistente en una serie de "working papers" escritos y publicados por profesores de la Escuela de Ciencias de la Educación de la Universidad Icesi con los que se busca apoyar la reflexión y la investigación en educación. Estos documentos pueden considerarse como "provisionales" y se publican bajo licencia Creative Commons (BY-NC-SA) para así estimular el debate académico entre diversos actores de la educación. Debate que puede tomar la forma de conversación, discusión, recuerdo, argumento, reflexión, sueño, escritura o conjetura; ojalá, frente a muchas tazas con café humeante.

Tal como lo plantea Marin-Garcia \& Garcia-Sabater (2010), los documentos de trabajo tienen como finalidad facilitar el acceso al debate académico de avances y resultados de trabajos de investigación, así como de fragmentos de investigación que posteriormente se pueden convertir en artículos científicos. De esta manera, estos trabajos académicos provisionales se vuelven citables y por tanto protegidos del plagio de ideas escritas y publicadas en versiones preliminares, sin menoscabo de ser divulgadas en los medios digitales ofísicos que su autor considere adecuados.

* Marin-Garcia, Juan \& Garcia-Sabater, José (2010). Los Working Papers al senvicio de la escritura productiva. Working Papers on Operations Management. 1(1). doi:10.4995/wpom.v1i1.790 


\title{
Impacto de los cursos de Comunicación Oral y Escrita (COE) entre estudiantes de la Universidad Icesi *
}

\author{
Investigador principal: Hoover Delgado Madroñero \\ Colaboradores: Juan Carlos Penagos y Alberto Rodríguez \\ Grupo de investigación IRTA, Universidad Icesi.
}

\section{Nota preliminar}

El presente informe corresponde a un primer avance de la investigación iniciada en 2014, que indaga sobre el impacto de los cursos de Comunicación oral y escrita I y II (COE) entre los estudiantes de la Universidad Icesi que han aprobado dichos cursos, ofrecidos en la etapa inicial de su formación universitaria. Encontrarán aquí información general sobre la propuesta, así como datos correspondientes a la primera fase de la investigación, que se centra en uno de los dos componentes sometidos a estudio: la lectura. Dificultades en el proceso llevaron a que se dejara la recolección y análisis del componente de escritura para una segunda fase.

Más allá de tratarse de una obra en marcha, posee el valor de documento-memoria sobre una investigación que busca convertirse en un proyecto institucional de largo plazo. De igual manera, se espera que los resultados totales sean publicados en medios y espacios académicos dentro y fuera de Icesi, y que contribuyan al ajuste de los cursos de COE, a la creación de un programa general del Departamento de Lenguaje centrado en el estudio de las competencias de lectura, escritura y oralidad, al desarrollo del proyecto de Valoración de la Universidad, y a la apertura de investigaciones similares con miras a enriquecer las perspectivas de análisis y enseñanza-aprendizaje del lenguaje.
Hoover Alfonso Delgado Madroñero es el director del Departamento de Lenguaje de la Escuela de Ciencias de la Educación (ECE), Universidad Icesi. Magister en Literatura Colombiana y Latinoamericana Candidato a Doctor en Literatura.

Juan Carlos Penagos Trujillo es profesor del Departamento de Lenguaje de la ECE, Universidad Icesi.

Alberto Rodríguez es profesor del Departamento de Lenguaje de la ECE, Universidad Icesi.

* Este documento de trabajo corresponde a un avance de la investigación registrada en el grupo IRTA: Impacto de los cursos de Comunicación Escrita entre los estudiantes de Icesi (casos semestres III, IV y V). Código del proyecto: CA061312. 


\section{RESUMEN}

El estudio tiene como objetivo dar cuenta de la incidencia, en términos de adquisición de competencias comunicativas en el contexto universitario, de los procesos de alfabetización académica que se llevan a cabo en los cursos de Comunicación oral y escrita (COE) de la Universidad Icesi. La muestra está integrada por 48 estudiantes de diversos programas profesionales que cursan materias de tercero a quinto semestre.

Es un estudio de tipo descriptivo, abordado desde la perspectiva de la didáctica de la lengua (Camps, 2011), siguiendo la línea de las investigaciones sobre prácticas de lectura y escritura en las universidades colombianas (Pérez Abril y Rincón Bonilla, 2013). En consecuencia, la metodología es de carácter mixto. Se hace uso de técnicas cualitativas (observación de prácticas e interacciones en el aula, revisión documental, entrevistas y grupos de discusión), y de técnicas cuantitativas (encuesta y pruebas diagnósticas), con el fin de recabar datos que permitan establecer los niveles de desempeño de escritura en que se encuentran los estudiantes, y caracterizar los elementos, dinámicas y aspectos del sistema didáctico (Camps, 2011) que determinan esos desempeños.

Dado que la perspectiva de abordaje es didáctica, se espera que los resultados sirvan de base a la reflexión sobre las prácticas de enseñanza y aprendizaje de la escritura académica en el contexto de la Universidad Icesi, y a la transformación y optimización de los procesos de alfabetización académica.

\section{Planteamiento del problema de investigación - justificación}

Recientemente se ha manifestado en los contextos universitarios un interés creciente por la comprensión y caracterización de las prácticas de lectura y escritura académicas de los estudiantes. Esto se debe a la necesidad de ofrecer una formación que permita a los estudiantes desempeñarse como agentes competentes en la comunicación profesional y científica; y a la constatación de que, en muchos casos, las competencias de los estudiantes distan mucho de permitirles acceder a los niveles de lectura y escritura que son exigidos por el medio académico.

En Colombia, recientes investigaciones se han dirigido a dar cuenta de los fenómenos descritos, y tienen por objetivo la caracterización en profundidad de los tipos de prácticas de lectura y escritura que realizan los estudiantes. Su enfoque se aparta de la simple medición de 
desempeños para tratar de identificar, desde una perspectiva didáctica, los factores propios de las culturas académicas que inciden en los procesos de aprendizaje de la lengua escrita por parte de los estudiantes. Un ejemplo en tal vía es la investigación “¿Para qué se lee y se escribe en la universidad colombiana? Un aporte a la consolidación de la cultura académica del país", de Pérez Abril y Rincón Bonilla (2013), cuyo acento reside en la comprensión de la forma en que variables tales como la cultura académica y la alfabetización académica inciden en los procesos de aprendizaje (Pérez Abril \& Rincón, 2013).

Los datos y experiencias derivados de investigaciones de tal naturaleza pueden servir de sustento para la planeación de procesos académicos que maximicen los resultados de las mediaciones didácticas destinadas a posibilitar el aprendizaje y el desarrollo de las competencias de escritura académica.

La Universidad Icesi no es ajena a este interés. Con el propósito general de indagar cuál es el estado real de las prácticas de lectura y escritura de sus estudiantes, nuestro estudio se enfoca, en primer lugar, en el análisis del impacto de los cursos de Comunicación oral y escrita (COE), que se ofrecen en los primeros semestres de la carrera, entre los estudiantes de los semestres $3^{\circ}$, $4^{\circ}$ y y $5^{\circ}$. Los cursos de COE, ofrecidos en los primeros semestres, se centran en el desarrollo de competencias comunicativas acordes con los requerimientos de la vida universitaria, y de las interacciones académicas que tienen como instrumento de mediación al texto escrito. De ahí que nuestro estudio busca específicamente determinar en qué nivel de apropiación de las competencias comunicativas se encuentran los estudiantes de los semestres posteriores al ciclo básico.

Adicionalmente, se pretende obtener datos que permitan reformulaciones pedagógicas y didácticas de la enseñanza de la escritura, los cuales sirvan para una optimización de los mismos cursos COE, y como guía para la aplicación de la política de lenguaje y comunicación de la Universidad. Por último, persigue construir un panorama de los factores externos a los cursos COE (entendidos como un primer momento de la alfabetización académica universitaria) que se encuentran en correlación con los niveles de desempeño (indicadores de la incidencia de los cursos COE) identificados durante la investigación, que tienen relación con las prácticas y sistemas de representaciones sobre lo escrito que integran la cultura académica de los estudiantes.

\section{Marco teórico y estado del arte}

\subsection{Aspectos relacionados con la alfabetización académica en escritura a ser abor- dados en este proyecto.}

Para trascender la evaluación de desempeños en procesos de escritura, y generar una caracterización de las prácticas de escritura de los estudiantes que permita comprender las razo- 
nes por las que se ubican en un nivel de desempeño, se pretende dar cuenta de los sistemas de representaciones que estos poseen con respecto a:

a) Los cursos de comunicación oral y escrita (alfabetización académica).

b) La escritura en el contexto de su formación académica por fuera de los cursos COE.

c) La escritura como práctica dentro del marco de la representación de sí mismos y de su proyecto de vida.

d) Los factores internos y externos a los cursos COE, propios de la cultura y las prácticas académicas de la Universidad Icesi, que tienden a reforzar los procesos de mejoramiento de la escritura o que tienden a presentarse como posibles causales de la neutralización o el estancamiento de dicho proceso.

e) Los factores relacionados con las historias de vida de estudiantes que refuerzan o afectan negativamente la apropiación y el mejoramiento de las prácticas de escritura académicas.

f) Los usos de la escritura que llevan a cabo los estudiantes y las finalidades a las que se destinan dichos usos.

La consecución de estos objetivos equivale a determinar cuál es el nivel de apropiación, por parte de los estudiantes, de los contenidos de las políticas de lenguaje y comunicación de la Universidad, y del enfoque semántico-comunicativo (Oviedo, 1997) de formación en lenguaje que subyace a los cursos de Comunicación Oral y Escrita.

Para ello, se indagará por los sistemas de representaciones de los estudiantes, y la forma en que estos se manifiestan en la práctica académica, con respecto a conceptos (categorías) fundamentales para la producción escrita tales como:

- Situación de comunicación, comunicación-significación (Oviedo, 2002).

- Dinámica enunciativa (Martínez, 2002, 2005).

- Significación y producción-negociación de sentido (Oviedo, 2002).

- Enunciado: actos de habla-significación (cohesión y morfosintaxis al nivel de la frase de acuerdo con el propósito y la intención del enunciador, y su representación del enunciatario en un contexto determinado) (Austin, 1962; Searle, 1962, 1969; Bustamante, 2001).

- Géneros discursivos (Charadeau, 2004).

- Tipologías textuales (Calsamiglia y Tusón, 2001). 
- Estructuras textuales: superestructuras (Van Dijk, 1983), macroestructuras: coherencia (Van Dijk, 1983), microestructuras: coherencia lineal y estructuras de párrafo (Serafini, 1994).

- Secuencias argumentativas (Adam, 1995).

Estos dispositivos de representaciones-prácticas serán abordados como elementos articuladores de los procesos de planeación y producción textual orientados a "expresar, describir, analizar, proponer y argumentar con la precisión, el orden, la economía verbal y la novedad que cada circunstancia y cada propósito requieren" (González, 2008, p.37), ideal que constituye la síntesis de lo que la Cartilla Docente titulada "El proyecto educativo de la Universidad Icesi y el aprendizaje activo" (González, 2008) presenta como objetivo en lo que respecta a la capacidad de comunicación de los estudiantes de la universidad.

A partir de este entramado conceptual se pretende describir el desempeño en la escritura de los estudiantes (indicador de la incidencia de los cursos COE), con base en el análisis de la forma en que se manifiesta en su escritura un conjunto de variables trabajadas y evaluadas en el marco de dichos cursos (Oviedo, 2012), entre las que se cuentan:

- La morfosintaxis: construcciones oracionales y morfología.

- La estructura de párrafos: entendida como una secuencia proposicional con una funcional textual específica, la cual puede ser sintetizada en una macroproposición o idea principal.

- La organización del texto extenso: entendida como la estructuración coherente de los diversos períodos o apartados del texto en función de una tipología textual específica y de un propósito y una intención comunicativos.

- Cohesión y coherencia: entendida como la interacción de las formas lingüísticas y los actos de habla-significación.

- El estilo: entendido como el conjunto de estrategias retórico-discursivas que sirven a la adecuación sociolingüística del texto a su auditorio y a la situación de comunicación. En esta dimensión de la escritura confluyen, según Oviedo (2012) los tonos, la creatividad, las figuras de dicción, el lexicón, la forma en que el estudiante dispone estratégicamente en sus textos las citas y referencias pertinentes para conseguir su propósito comunicativo, y la adecuación a los formatos de citación y referencia.

- La puntuación: entendida como el conjunto de operaciones de segmentación de los textos que sirve a la especificación de las relaciones entre actos de habla-significación (proposiciones expresadas bajo la forma de frases) de las cuales depende la generación del sentido. 


\subsection{Precedentes}

Entre los precedentes para este estudio se encuentra un conjunto de estudios sobre la escritura y la lectura en las universidades colombianas que se encuentran compilados en el libro Para qué se lee y se escribe en la universidad colombiana. Un aporte a la cultura académica del país (Pérez-Abril y Rincón-Bonilla, 2013). En general, se encontró que los estudiantes presentan falencias en lo que respecta a sus competencias de lectura y escritura académicas, y que el desarrollo de las competencias comunicativas de los estudiantes para que se inserten adecuadamente en la cultura académica (Arias y Agudelo, 2010) requiere de procesos de alfabetización académica continuos y sistemáticos (Arias y Agudelo, 2010; Pérez-Abril y Rincón Bonilla, 2013).

Esto ocurre porque los géneros discursivos, las tipologías textuales y los usos de los textos que se dan en la universidad difieren de los que caracterizan a la educación secundaria. Los estudiantes deben apropiarse los sistemas conceptuales de la textualidad e insertarse en las prácticas discursivas de la universidad, y para esto necesitan participar de nuevos procesos de alfabetización, adecuados a lo que se espera de ellos durante el desarrollo de sus estudios profesionales.

Otros autores, como Carvajal y Ulloa (2010) presentan investigaciones que sirven de base metodológica al estudio que aquí se presenta. Carvajal y Ulloa, se evaluaron las competencias de lectura y escritura de los estudiantes de Comunicación Social de la Universidad del Valle. Los instrumentos utilizados fueron una encuesta y pruebas de lectura y escritura. El diseño de las pruebas se basó en la perspectiva discursiva y enunciativa (privilegiada en el conjunto de las investigaciones revisadas), y en aspectos básicos de lingüística textual. Se evaluó si los estudiantes eran capaces de:

- Identificar los enunciadores presentes en el texto leído, como una condición necesaria para su comprensión (prueba de lectura).

- Identificar todos los puntos de vista presentes en el texto empleado para la evaluación de los estudiantes (prueba de lectura).

- Representar, por escrito, la tesis y los argumentos del autor del texto leído (prueba de escritura).

- Representar los enunciadores incluidos en el texto escrito por ellos (prueba de escritura).

- Presentar de manera argumentada su punto de vista personal sobre el texto leído o sobre el tema del mismo (prueba de escritura).

- En la prueba de escritura, se exigía que los estudiantes presentaran dos puntos de vista 
y dieran cuenta de una organización en regla de su argumentación a partir de una problemática tomada de un artículo de opinión. Se evaluó:

* La capacidad para identificar y formular en un resumen la tesis y los argumentos del autor del texto leído.

* El reconocimiento de los puntos de vista considerados por el autor del ensayo.

* La manera como los estudiantes representaban en su resumen los diferentes enunciadores reconocidos en la lectura.

* La capacidad para presentar de manera clara, coherente y sustentada su punto de vista personal sobre el texto objeto de la prueba o sobre el tema desarrollado en el mismo (Carvajal y Ulloa, 2010, p.51).

Lo que muestra este estudio es que no todos los estudiantes presentan el mismo nivel de adecuación a las exigencias de la escritura y la lectura académicas, y que estas diferencias dependen, según el cruce de datos entre la encuesta y las pruebas, de factores no solo escolares sino también sociales y relativos a los consumos culturales y los hábitos de los sujetos. También se evidencia que a lo largo de la carrera universitaria se presentan algunos cambios y mejoramientos en la lectura y escritura de los estudiantes. Sin embargo, siguen siendo necesarias la planeación e implementación de procesos de alfabetización académica sistemáticos, orientados a acortar la brecha entre los que presentan buenos desempeños y los que presentan mayores dificultades.

A continuación se presenta sucintamente una revisión conceptual de los principales aspectos de la lingüística textual y la dinámica enunciativa que serían explorados a través de los desempeños de los estudiantes en la escritura.

\subsection{Criterios de la textualidad.}

\subsubsection{Nivel Superestructural de los textos.}

El análisis de las superestructuras parte del hecho de que las mismas "no se definen con relación a oraciones o secuencias aisladas de un texto, sino para el texto en su conjunto o para determinados fragmentos de este" (Van Dijk, 1983: 142-143). Se debe a que las superestructuras tienen un carácter global. Es por medio de la identificación de superestructuras que los lectores son capaces de adjudicar un texto a una determinada tipología textual históricamente determinada en el seno de un género discursivo.

Según Van Dijk (1983: 143) "las superestructuras no solo permiten reconocer otra estructura más, especial y global, sino que a la vez determinan el orden (la coordinación) global de 
llama unidades, que responden a una "categoría determinada" y "están vinculadas con las partes del texto previamente ordenadas" (Van Dijk, 1983: 143).

En consecuencia, la superestructura sería "una especie de esquema al que el texto se adapta" (Van Dijk, 1983: 143). Este esquema, propio de una tipología textual, es independiente del contenido del texto mismo, y de las estructuras "lingüísticas", y por tal motivo Van Dijk (1983: 143) afirma que suele tener un carácter convencional. Equivale a decir, con respecto a las superestructuras, que "la mayoría de hablantes de una comunidad lingüística las conoce o reconoce" (Van Dijk, 1983: 143).

En consecuencia, las superestructuras se identifican como "esquemas abstractos que establecen el orden global de un texto y que se componen de una serie de categorías, cuyas posibilidades de combinación se basan en reglas convencionales" (Van Dijk, 1983: 144). En el caso del corpus a ser analizado en nuestro trabajo, el análisis deberá centrarse, por tanto, en identificar cuáles son las categorías (entendidas como partes de la estructura global) que integran el texto.

\subsubsection{Nivel Macro-estructural}

Van Dijk (1980: 219) relaciona las macroestructuras con los tópicos de conversacióndiscurso que dan unicidad al texto, y a partir de los cuales se establecen tanto la coherencia lineal (entre oraciones) y la coherencia global entre secuencias. Una macroproposición sería la proposición que, en el plano semántico, une otras proposiciones presentes en el texto y garantiza la coherencia entre ellas. En el siguiente ejemplo presentado por Van Dijk (1980: 220): Las casas en la ciudad estaban en ruinas, y muchos parados vagaban por las esquinas, ambas cláusulas son sintáctica y semánticamente aceptables, pero lo que asegura que sean leídas como correspondientes a un mismo tópico o referencia (la ciudad) y como partes articuladas de la producción de sentido con respecto a ese tópico, es el hecho de que pueden ser sintetizadas semánticamente en una tercera proposición, que garantiza la coherencia del período: La ciudad estaba en decadencia.

En este caso, la coherencia que se presenta es lineal, y en la coherencia global la operación es similar, solo que tiene lugar articulando las macroproposiciones que sintetizan cada secuencia de un texto.

El principio macroproposicional de la producción de la coherencia semántica se confirma cuando se considera lo que acontece en ausencia del tópico que garantice la articulación entre cláusulas o proposiciones, como en el siguiente caso presentado por Van Dijk (1980: 220): Juan nació en Manchester, y vamos a ir a la playa. Aunque ambas cláusulasproposiciones son correctas sintácticamente y establecen su propia referencia, dicha referencia no es compartida y no es posible una síntesis semántica entre ambas. 
Pero la referencia no basta para que se garantice la coherencia textual. En el siguiente ejemplo, también propuesto por Van Dijk (1980: 221), todas las proposiciones que integran la secuencia comparten algún referente entre sí y podrían establecerse macro-proposiciones sintetizando siempre dos, pero nunca las tres: Compré esta máquina de escribir en Nueva York. Nueva York es una gran ciudad de USA. Las grandes ciudades a veces tienen serios problemas financieros... Aquí es imposible articular las tres proposiciones como parte del desarrollo de un mismo tema o tópico. Es el tópico, en compañía de las referencias compartidas, lo que permite que se establezcan nexos macroproposicionales, es decir, que se articulen semánticamente las distintas proposiciones y secuencias que componen un texto. Es a esta articulación, dependiente de la existencia de macroestructuras, a lo que se denomina coherencia textual.

El principio de la coherencia se encuentra en la base de la progresión temática de los textos, la cual a su vez equivale al encadenamiento de las ideas por medio de la sucesión entre el tema (la información ya conocida) y el rema (la información nueva), para que se alcance el propósito comunicativo. Todo lo anterior según un plan estratégico vinculado a la intención que subyace al proyecto de enunciación del texto.

Es necesario tener en cuenta que las macroestructuras son de tipo semántico, y que la construcción semántica del sentido global del texto es posible porque, según Van Dijk (1980: 222), las frases y las cláusulas del texto, centradas en el desarrollo semántico y cognitivo de la textualidad, expresan proposiciones y propiedades de proposiciones. Es decir, las macroestructuras, integradas por macroproposiciones, no equivalen a oraciones reales, sino a "propiedades de nivel superior de secuencia de proposiciones" (Van Dijk, 1980: 222). Estas propiedades pueden ser del tipo de "relaciones entre argumentos, predicados, operadores modales, etc." (Van Dijk, 1980: 222).

Cómo ha sido indicado por medio de los ejemplos provistos por Van Dijk (1980), un texto construye significado a partir de un dispositivo proposicional. La coherencia linealsecuencial, de la que es producto el significado lineal-secuencial, y la coherencia global, que es el resultado del esquema macroproposicional, son garantizadas por las relaciones que el plan de enunciación-escritura establece entre niveles proposicionales.

Van Dijk (1980) reconoce dos niveles proposicionales. El primero, vinculado a la coherencia lineal, recibe el nombre de nivel microtextual, y consiste en "la estructura semántica de la secuencia de frases" (Van Dijk, 1980: 213). Sobre la base de las relaciones micro se construyen, a partir de "conjuntos de proyecciones semánticas" (Van Dijk, 1980: 213), las relaciones macroproposicionales. Según Van Dijk (1980: 213), para identificar el orden macroproposicional de un texto es necesario aplicar un conjunto de operaciones de reducción semántica sobre los significados que el orden construye lineal y localmente en el nivel de las secuencias de proposiciones que lo integran. De esta manera: 
"Un cierto número de proposiciones pueden ser substituidas por una (macro) proposición que «subsuma» la información más detallada en un nivel más global de representación. Es esta macroproposición la que da cuenta del hecho de que la secuencia original de proposiciones forma una unidad semántica en relación con el nivel de la macroproposición" (Van Dijk, 1980: 213).

Por tanto, para describir una estructura textual, se hace necesario establecer cuáles son las secuencias que subyacen a la estructura macroproposicional de un texto.

\subsection{Secuencias textuales y nivel microestructural.}

Para Calsamiglia y Tusón (2001: 265), quienes a su vez se basan en la propuesta de análisis de Adam (1992), las unidades constituyentes del texto son las secuencias. Una secuencia está "constituida por paquetes de proposiciones (P: proposiciones), a su vez constituidas por $\mathrm{n}$ proposiciones (p: microproposiciones)".

Siguiendo a Adam (1992), se puede afirmar que las secuencias responden a estructuras prototípicas, es decir, "a tipos relativamente estables de combinación de enunciados, dotados de una organización reconocible por su estructura jerárquica interna (esquema) y por su unidad compositiva (plan)”. Es por ello que, en términos analíticos, las secuencias son concebidas como "un modo de segmentación que permite articular la complejidad textual, proporcionando una vía para (...) establecer tipos de texto" (Calsamiglia y Tusón, 2001: 266).

Para Adam (1999), "la operación de ligazón de las proposiciones lleva a dos grandes tipos de paquetes de proposiciones: unidades textuales no tipificadas que llamaremos períodos y unidades a menudo más complejas y tipificadas que llamaremos secuencias". Los períodos se distinguen de las secuencias, además, por el hecho de que son de menor amplitud y porque las secuencias son, dada su convencionalidad, "estructuras periódicas complejas, y sobre todo predefinidas, de agrupamientos de proposiciones" (Adam, 1999).

Adam complementa la definición de los períodos cuando afirma que son unidades rítmicas del texto, que replican (y modifican) en el plano de la escritura la segmentación propia de la oralidad, y mantienen entre sí relaciones de dependencia marcadas morfosintácticamente en el plano de la organización textual. Las secuencias agruparían períodos o estarían constituidas por estos. Los períodos, según Adam (1999) pueden presentarse como agrupamientos rítmicos de proposiciones "mediante toma de fonemas/grafemas, lexemas, sintagmas enteros" o como agrupamientos vinculados que dependen de la función de organizadores y conectores para articular proposiciones en términos de coherencia local y lineal.

Dentro de este modelo, lo que Van Dijk ha denominado macroproposiciones puede ser entendido como "agrupamientos tipificados de proposiciones propias" (Adam, 1999) de los diversos tipos de secuencias. Las secuencias prototípicas propuestas por Adam (1992, citado 
por Calsamiglia y Tusón, 2001: 265) son la narrativa, la descriptiva, la argumentativa, la explicativa y la dialogal. Un texto concreto sería una combinatoria de algunos (o incluso todos) estos conjuntos de secuencias.

\subsection{Situación de enunciación}

Según Martínez (2002), el proceso de la comunicación discursiva se realiza como práctica social" y "través de los enunciados en ella construidos se ponen en escena las diferentes imágenes de escritor y de lector". Lo que se genera en la interacción de enunciados son sujetos discursivos (Martínez, 2002). Los enunciados están inscritos en un "género discursivo particular o una práctica social concreta” (Martínez, 2002), y a través de la observación de la dinámica de los intercambios se "pueden establecer los momentos en que se produce una alteridad, es decir, el cambio entre los sujetos que intervienen en una situación discursiva" (Martínez, 2002).

La situación de enunciación se basa en el establecimiento de una dinámica dialógica entre dos o más sujetos que se construyen en la interacción y a través de los enunciados en un contexto de situación. En esta dinámica, el contexto se integra determinando las posibilidades de la comunicación y los géneros discursivos pertinentes, y distribuyendo roles socioinstitucionales a los cuales habrán de responder los sujetos.

La situación de enunciación está integrada por sujetos que actúan como locutores, pero que gracias al enunciado devienen imágenes y sujetos discursivos en la enunciación. En el discurso se establecen diversas formas de organizar las relaciones intersubjetivas entre el locutor, los enunciadores, las voces en que estos enunciadores se apoyan, y lo enunciado o referido (tercero).

Esto ocurre porque en el contexto de una concepción bajtiniana del enunciado "las fuerzas sociales enunciativas se construyen en relación con la imagen del Locutor, en términos de Enunciador, con la imagen del interlocutor, en términos de Enunciatario, y, la imagen del Tercero o Voz ajena, en términos de Lo Enunciado" (Martínez, 2005: 74). Estos tres actores discursivos, el Enunciador, el Enunciatario y lo Enunciado, son "los sujetos discursivos responsables de la dinámica enunciativa en un discurso” (Martínez, 2005: 74).

La dinámica enunciativa se establece por medio de las operaciones de alejamiento o acercamiento entre los sujetos discursivos. En tal sentido, según Martínez (2005: 75), en todos los discursos "se constituye una situación de enunciación específica de la cual dan cuenta, precisamente, las formas de manifestación textual que adquiere el texto o el enunciado". Dichas formas de manifestación textual dependen de, y determinan a las imágenes de los sujetos discursivos que se producen en la enunciación. Por tanto, la enunciación de los discursos no solo se orienta socialmente hacia el interlocutor, de quien, señala Martínez (2005: 76), "se construye una imagen de Enunciatario en términos de aliado, testigo o enemigo", sino que 
además se orienta hacia el Tercero, que corresponde a "la voz ajena que se alaba o critica, cuya relación es expresada a través de actos de habla que indican agresión, amenaza, defensa, consideración, aprecio o admiración" (Martínez, 2005: 76). Esto equivale a afirmar que los actos de habla (Austin, 1962; Searle, 1962, 1999), en tanto que elementos integradores de la enunciación, son los agentes y manifestaciones de una actitud apreciativa con respecto a lo enunciado.

Es por ello que Martínez (2005: 78) indica que "el Enunciador se construye en relación (...) con una doble orientación social: el valor de jerarquía y el grado de intimidad con el Tercero (héroe), e igualmente la valoración social con respecto al Enunciatario". De esta manera se manifiesta no solo la doble orientación social de la enunciación, sino el hecho de que en esta se expresan relaciones de fuerza entre los sujetos discursivos, que son, en última instancia, relaciones de poder que replican las relaciones que estructuran las jerarquías y los valores del sistema social y el campo de producción en el que el discurso encuentra su razón de ser.

Por otra parte, la importancia del discurso ajeno en la dinámica enunciativa, estudiada por Bajtin, consiste en el hecho de que los actos de habla se complementan con los discursos referidos (directo e indirecto) para constituir las diversas perspectivas de la relación entre el Locutor que se presenta como Enunciador y los restantes sujetos discursivos. Es a partir de esta dinámica enunciativa que: "La realización del enunciado a través de sus formas de manifestación, de sus maneras de decir, dará cuenta de la actitud valorativa, la postura social, que tiene el autor hacia el contenido, a través de las relaciones de fuerza enunciativa construidas entre el Enunciador y el Tercero, y con respecto al Enunciatario. La manera como el autor percibe el contenido o el Tercero se mostrará a través del uso de narraciones objetivas, descripciones, formas apelativas o formas de autoexpresión" (Martínez, 2005: 78).

De esta manera se construyen las que Martínez denomina orientaciones sociales de la argumentación, en las que se presenta una relación dinámica entre las dimensiones discursivas del pathos (que solía atribuirse en la retórica clásica al Enunciatario), el ethos (que solía atribuirse al Enunciador), y la ratio del Saber -o logos- (que solía identificarse con el Tercero). Según Martínez (2005: 88), estas tres dimensiones se manifiestan en todos los actores que intervienen en la situación como sujetos discursivos. Esto se evidencia en el hecho de que, al argumentar, "el Enunciador puede convocar características que muestran una imagen relativa a su ethos (honesto), a su pathos (amable) o a su 'ratio' (prudente)" (Martínez, 2005: 88), al tiempo que convoca "simultáneamente, una relación parecida con el Enunciatario" (Martínez, 2005: 88-89). Es decir, en el discurso los sujetos discursivos se constituyen a partir de la articulación de tres dimensiones: ética, emotiva y cognoscitiva.

Dependiendo de las instancias del discurso, el plan estratégico y las intenciones del Locutor en el texto puede privilegiarse una orientación cognoscitiva (centrada en la ratio), emotiva (centrada en el pathos), o ética (centrada en el ethos). 
En este contexto, los actos de habla, en tanto que actos discursivos, se encuentran "en relación con el tipo de interacción social enunciativa que se busca mostrar" (Martínez, 2005: 95). La interacción enunciativa se crea a partir de la actualización de las jerarquías y los grados de intimidad que el Locutor devenido Enunciatario establece con respecto al Tercero. Así mismo, son determinantes de la configuración de una situación enunciativa la intención y los propósitos del locutor y las tonalidades que dé a su enunciado.

La dinámica enunciativa manifiesta un carácter polifónico en la medida en que la presencia del Tercero como Voz Ajena, y la relación intersubjetiva y dialógica entre Enunciador y Enunciatario genera "un desdoblamiento del sujeto en varias voces" en el que el Locutor "actúa como responsable del enunciado (el cual no siempre coincide con el autor empírico...)” (Martínez (2005:98). Sin embargo, en los enunciados aparecen, en función de la referencia a la voz ajena propia de la dialogicidad del discurso, varios "locutores responsables de enunciados anteriores, diferentes al enunciado que los refiere” (Martínez, 2005: 98). Tales enunciados referidos (discurso ajeno, en términos de Bajtin) corresponden a las diversas voces textuales que se manifiestan en el discurso. Con respecto a las voces textuales y su función en el análisis de la dinámica enunciativa, indica que:

"La manera como se presentan los enunciados en el discurso referido ofrece indicios para identificar el tipo de relación que se establece entre los locutores: entre el locutor responsable del enunciado que reporta y el locutor responsable del enunciado reportado. Los estilos directo, indirecto e indirecto libre, así como las comillas de palabras, son indicios de esta relación polifónica” Martínez (2005:99).

Sumado a lo anterior, Reyes (2002) en una perspectiva pragmática y metapragmática del análisis de los actos de habla y el lenguaje en uso, entiende por enunciadores "los agentes de los actos o los puntos de vista presentados en el discurso".

\section{Objetivos.}

Objetivo General.

Establecer la incidencia que los cursos COE tienen en las prácticas de escritura de los estudiantes de tercero, cuarto y quinto semestre de la Universidad Icesi.

Objetivos Específicos.

Identificar los factores internos y externos a los cursos COE, propios de la cultura y las prácticas académicas de la Universidad Icesi, que tienden a reforzar los procesos de mejoramiento de la escritura, o a presentarse como posibles causales de la neutralización o el estanca16 miento de dicho proceso.

Identificar los factores relacionados con las historias de vida de estudiantes, y su relación 
con la lectura y la escritura, que refuerzan o afectan la apropiación y el mejoramiento de las prácticas de escritura académica.

Caracterizar los usos de la escritura que llevan a cabo los estudiantes y las finalidades a las que se destinan dichos usos.

\section{Metodología y planificación general del proyecto.}

Por cuanto se pretendía dar cuenta de la incidencia de los cursos COE en las representaciones y prácticas de escritura de los estudiantes de la Universidad Icesi, el carácter descriptivo de nuestro estudio exigió un abordaje principalmente cualitativo, basado en técnicas de análisis cuantitativo que hicieran posible la medición de las variables a ser evaluadas a partir de la aplicación de una batería de pruebas de escritura. Se aplicaron tres pruebas en diferentes momentos del semestre, adecuadas a las tipologías textuales y a los temas vistos en diversos programas académicos que demandaran solución a situaciones y problemas de comunicación.

Durante los análisis, la variable dependiente fue el nivel de desempeño de escritura (que da cuenta de la incidencia de los cursos COE a partir de la apropiación y aplicación de los conceptos por parte de los estudiantes). Las variables independientes fueron los aspectos de la evaluación de los cursos COE indicados anteriormente, junto con los conceptos de lingüística textual y del enfoque semántico comunicativo (Oviedo, 1997) con los que estos se relacionan.

\section{Resultados}

\subsection{Encuesta.}

El estudio se llevó a cabo a lo largo de dos semestres, con una muestra conformada por 48 estudiantes que agrupaba a todas las carreras que ofrece la Universidad. Para la recolección de los datos se hizo uso de un instrumento mixto, con el formato de encuesta, en el que se indagó por:

a) el conocimiento de los estudiantes con respecto a las diversas tipologías textuales de uso académico y sus estructuras y funciones.

b) el conocimiento de los estudiantes con respecto a los rudimentos de la lingüística textual, y con respecto a la situación de comunicación-enunciación, que se requieren para comprender los fenómenos textuales, su funcionamiento, y sus funciones en términos comunicativos y pragmáticos.

c) las prácticas relativas a la planeación de los textos y su relación con lo que, según los programas de los cursos COE, han aprendido los estudiantes a lo largo de estos. 
d) las prácticas de retroalimentación y corrección de los textos.

e) los tiempos y espacios en que se distribuyen las prácticas de escritura de los estudiantes

El formato de la encuesta estaba encabezado por una definición de los aspectos de la textualidad por cuya apropiación se iba a indagar. Por tanto, el diligenciamiento del instrumento empezaba por un recordatorio teórico, de modo que no existiera la posibilidad de que los estudiantes se confundieran, o no recordaran, de qué se trataba aquello por los que se les estaba preguntando.

Un segundo instrumento consistió en una prueba diagnóstica en la que se indaga por la manera en que, durante el proceso de la lectura crítica, los estudiantes hacen uso de sus saberes textuales, discursivos y relativos a la argumentación. Allí se identificarían los aspectos más relevantes de la producción de sentido, tales como los elementos propios de la situación de comunicación-enunciación, las macroestructuras del texto, y las funciones argumentativas de las macroproposiciones del mismo.

Por último, se buscaba indagar el desempeño de los estudiantes en tanto que escritores, solicitándoles la producción de un artículo-ensayo corto a través del cual ofrecieran su punto de vista a propósito del tema de los textos expertos consignados en el formato de diagnóstico. Debido a la reticencia constante de los estudiantes a exponer su producción escrita no se pudieron recolectar suficientes datos correspondientes a este tópico, por lo que la investigación debió posponer la información sobre el aspecto para la nueva fase del proyecto, que se adelantará en el año 2015, buscando reestructurar la dinámica de participación de los sujetos participantes en el estudio. Para ello, se planea hacer análisis de los textos correspondientes a cursos específicos en los que los estudiantes realizan entregas de trabajos, y no de textos pedidos dentro de la consigna del estudio.

En cuanto al componente de lectura, se ha podido observar que los estudiantes poseen un conocimiento de las estructuras textuales básicas, y que identifican estructuras argumentativas básicas. No obstante, los resultados de la prueba de diagnóstico parecen indicar que este nivel de comprensión no es suficiente para dar cuenta de estructuras argumentativas más complejas, como las correspondientes al modelo de Toulmin, que tradicionalmente se trabaja en el curso de COE II.

En general, los datos muestran que los estudiantes se representan su paso por COE como una oportunidad para aprender a pensar el texto como estructura organizada, con base en estructuras más sencillas como el párrafo. Haría falta acceder a su escritura para confirmar si efectivamente este tipo de aprendizaje tiene una incidencia positiva en sus desempeños como escritores académicos.

\subsection{Estructura de la encuesta}


La primera parte de la encuesta estuvo dirigida a establecer cuáles eran las tipologías textuales más usadas en las prácticas de escritura de los cursos a los que atendían los estudiantes que integraron la muestra, correspondiente a las diferentes carreras de la universidad.

En la tabla 1 se presentan los resultados correspondientes a la distribución de dichas tipologías textuales según su incidencia en las prácticas académicas.

Tabla 1

\begin{tabular}{|l|c|c|}
\hline Apuntes de clase & $\mathbf{1 4}$ & $\mathbf{4 7 \%}$ \\
\hline Síntesis y resúmenes a partir de textos expertos & $\mathbf{8}$ & $27 \%$ \\
\hline Reseñas & $\mathbf{3}$ & $10 \%$ \\
\hline Ensayos críticos & $\mathbf{2}$ & $7 \%$ \\
\hline Artículos académicos & $\mathbf{2}$ & $7 \%$ \\
\hline Respuestas a cuestionarios & $\mathbf{0}$ & $0 \%$ \\
\hline Otros & $\mathbf{0}$ & $0 \%$ \\
\hline
\end{tabular}

Coincidiendo con los resultados de los antecedentes de la investigación, se observa que los apuntes de clase ocupan un lugar privilegiado entre los textos de mayor uso y consulta por parte de los estudiantes. Tal tipo de texto sirve a la recolección de información, a la síntesis de macroproposiciones de los temas tratados, al almacenamiento de la información que el estudiante considera relevante de acuerdo con el contrato didáctico de la clase y las dinámicas de evaluación de la misma.

Se observa que la síntesis y el resumen, aunque ocupan el segundo lugar, no tuvieron relación con los apuntes de clase ni con las reseñas, de las que sin duda son parte fundamental. Asimismo, llama la atención la poca la presencia de los ensayos críticos y los artículos académicos. Es manifiesto el uso de la escritura con fines ligados a la tarea de clase y la ausencia de escritura con fines científicos o de pensamiento crítico-reflexivo. Dada la limitación de la muestra que integró el estudio, dicho resultado servirá para ser confrontado con la segunda parte a ser realizada durante el año 2015.

Es importante señalar que los cursos de COE responden, precisamente, a la necesidad de aprender a tomar, realizar síntesis, y componer reseñas, tipologías textuales de mayor incidencia entre la población estudiada.

Un 40\% de la muestra manifestó que los cursos COE les habían brindado formación pertinente para la escritura de estas tipologías textuales, mientras que un 53\% manifestó que, aunque esta formación no había sido explícita, se habían ofrecido los rudimentos necesarios para la identificación y producción de las tipologías textuales mencionadas. Con ello se con- 
firmaría la pertinencia de los cursos COE y su adecuación a la demanda de competencias de escritura que la cultura académica de la Universidad Icesi desarrolla entre sus estudiantes.

Se presenta a continuación la distribución de los datos correspondientes al conocimiento que poseen los estudiantes de los sistemas de representaciones de la lingüística textual y discursiva que sirven a la comprensión de las dinámicas de la comunicación escrita.

Tabla 2

\begin{tabular}{|l|c|c|}
\hline Acción dentro de la práctica & $\begin{array}{c}\text { Número de } \\
\text { estudiantes }\end{array}$ & $\mathbf{1 7}$ \\
\hline $\begin{array}{l}\text { Identifica el género discursivo al que corresponden los } \\
\text { textos }\end{array}$ & $\mathbf{1 2}$ & $57 \%$ \\
\hline $\begin{array}{l}\text { Identifica la tipología textual a la que corresponden los } \\
\text { textos }\end{array}$ & $\mathbf{1 2}$ & $40 \%$ \\
\hline $\begin{array}{l}\text { Identifica la superestructura que corresponde a la } \\
\text { tipología textual }\end{array}$ & $\mathbf{2 8}$ & $93 \%$ \\
\hline \begin{tabular}{l} 
Identifica el tema del texto \\
\hline Identifica la intención comunicativa
\end{tabular} & $\mathbf{2 3}$ & $77 \%$ \\
\hline $\begin{array}{l}\text { Identifica el acto de habla en el que se condensa el } \\
\text { propósito del enunciador con respecto a sus enunciatarios }\end{array}$ & $\mathbf{7}$ & $23 \%$ \\
\hline $\begin{array}{l}\text { Distingue entre el autor, el locutor, y el enunciador } \\
\text { principal del texto }\end{array}$ & $\mathbf{2 1}$ & $70 \%$ \\
\hline $\begin{array}{l}\text { Identifica las proposiciones en las que se condensan los } \\
\text { puntos de vista que desarrollan el tema }\end{array}$ & $\mathbf{1 0}$ & $33 \%$ \\
\hline $\begin{array}{l}\text { Identifica las diferentes secuencias textuales (narrativa, } \\
\text { dialogal, descriptiva, expositiva-explicativa, } \\
\text { argumentativa) que integran el texto y sus funciones al } \\
\text { interior de la estrategia discursiva del autor }\end{array}$ & $\mathbf{2 1}$ & $\mathbf{2 0}$ \\
\hline $\begin{array}{l}\text { Identifica las proposiciones que sintetizan cada período } \\
\text { del texto (macroproposiciones) }\end{array}$ & $\mathbf{9}$ & $\mathbf{1 7}$ \\
\hline $\begin{array}{l}\text { Identifica la relación existente entre las } \\
\text { macroproposiciones en las que se puede sintetizar cada } \\
\text { período del texto }\end{array}$ & $\mathbf{7}$ & $23 \%$ \\
\hline $\begin{array}{l}\text { Establece previamente una tesis para los textos que } \\
\text { escribe }\end{array}$ & $\begin{array}{l}\text { Identifica los datos que sustentan la tesis en los textos que } \\
\text { lee }\end{array}$ & $57 \%$ \\
\hline
\end{tabular}




\begin{tabular}{|l|c|c|}
\hline $\begin{array}{l}\text { Selecciona los datos más pertinentes para el sustento de } \\
\text { las tesis de sus textos }\end{array}$ & $\mathbf{2 0}$ & $\mathbf{6 7 \%}$ \\
\hline $\begin{array}{l}\text { Identifica el garante que permite el paso "lógico" entre los } \\
\text { datos y las tesis de los textos que lee }\end{array}$ & $\mathbf{1 3}$ & $43 \%$ \\
\hline $\begin{array}{l}\text { Selecciona sus datos, y establece un garante de la relación } \\
\text { entre estos y las tesis de sus textos, de acuerdo con la } \\
\text { lógica interna del discurso profesional dentro del cual está } \\
\text { escribiendo }\end{array}$ & $\mathbf{1 5}$ & $50 \%$ \\
\hline $\begin{array}{l}\text { Identifica los soportes o respaldos que sustentan a los } \\
\text { garantes en los textos que lee }\end{array}$ & $\mathbf{8}$ & $27 \%$ \\
\hline $\begin{array}{l}\text { Establece, de manera explícita, respaldos o soportes para } \\
\text { sus textos }\end{array}$ & $\mathbf{1 5}$ & $50 \%$ \\
\hline $\begin{array}{l}\text { Identifica las reservas que se introducen en el desarrollo } \\
\text { de los textos que lee, y su función argumentativa }\end{array}$ & $\mathbf{9}$ & $30 \%$ \\
\hline $\begin{array}{l}\text { Identifica las posibles reservas que puedan afectar la } \\
\text { validez general de la tesis de sus textos, y las integra a su } \\
\text { plan de escritura }\end{array}$ & $\mathbf{1 1}$ & $37 \%$ \\
\hline $\begin{array}{l}\text { Durante sus lecturas, identifica argumentos no válidos } \\
\text { Distingue entre falacias y paralogismos }\end{array}$ & $\mathbf{1 4}$ & $47 \%$ \\
\hline $\begin{array}{l}\text { Al planear sus textos evalúa los argumentos y datos de } \\
\text { modo que no introduzca falacias ni paralogismos en sus } \\
\text { argumentaciones }\end{array}$ & $\mathbf{9}$ & $30 \%$ \\
\hline
\end{tabular}

Aquí se presentó un fenómeno interesante. Si bien la encuesta estaba encabezada por una definición de los aspectos de la textualidad que se indagaban, la misma arroja datos contradictorios con respecto a la primera parte del documento. Por ejemplo, apenas el $40 \%$ de los estudiantes manifiesta identificar la tipología textual de los textos leídos en sus cursos, y la superestructura con la que esta tipología textual se relaciona. Se evidencia así un vacío u olvido de trabajo más estructurado de los textos, frente a aquel que se promueve en los cursos COE, los cuales, en línea con los estudios de Serafini, Carlino y Cassani, trabajan la noción, usos y funciones del párrafo como unidad semántica básica.

Así mismo, llama la atención que el 93\% de los estudiantes manifiesta que identifican los temas de los textos con los que entran en relación como parte del desarrollo de sus cursos, pero apenas un 30\% identifica las macroproposiciones que sintetizan cada parte del texto, y un $23 \%$ identifica la relación entre estas, de las que depende la progresión temática. Ello podría deberse a la falta de conocimiento de los estudiantes de la teoría lingüística textual, pero también puede estar dando cuenta de unas representaciones problemáticas acerca de qué es el tema y cómo se construye. Para confirmar cuál es el caso, se deben analizar los resultados de la prueba diagnóstica de lectura y escritura que se plantea como segundo instrumento del estudio. 
Así mismo, se encontraron contradicciones en los datos referentes al modelo argumentativo de Toulmin, uno de los modelos centrales de la formación en COE II. Cabría suponer que, si un modelo tal establece relaciones de dependencia y derivación entre sus partes, la identificación de cada una de ellas sería más o menos constante, y que su planeación para la escritura de textos correspondería a la claridad con que los estudiantes los identifican en el material que leen.

Esto no ocurre así. Mientras el 27\% de los estudiantes identifica el respaldo en la red argumentativa de los textos que lee, un 50\% los establece de manera explícita para sus escritos. Así mismo, apenas un 43\% identifica el garante que permite el paso "lógico" entre los datos y las tesis de los textos leídos, mientras que un 50\% selecciona sus datos, y establece un garante de la relación entre datos y tesis, de acuerdo con la lógica interna del discurso profesional que elabora.

Según esto, la escritura de los estudiantes sería más monitoreada que sus lecturas. No obstante, cuando se indaga por la categoría de "dato" se encuentra que el 60\% de los estudiantes identifica los datos que sustentan la tesis en los textos que lee, y es igual el porcentaje de los estudiantes que, en el momento de la escritura, selecciona los datos más pertinentes para el sustento de las tesis.

Los resultados anteriores, así como la entrevista personal y la observación de clases nos permiten colegir que el énfasis puesto en las nociones de dato y tesis genera una apropiación limitada del modelo de Toulmin por parte de los estudiantes. Sin embargo, esta apropiación sería suficiente para las exigencias de orden argumentativo y de escritura académica que se hacen a los estudiantes a lo largo de sus carreras.

\subsection{Planeación de los textos.}

En lo que respecta a la planeación de los textos: 53\% de los estudiantes manifiesta que planifican sus textos siguiendo los modelos de composición de párrafos antes señalados, en lugar de un modelo macroproposicional y superestructural; por el contrario, 43\% manifiesta que no lleva a cabo ninguna planeación. En general, dicha planeación se hace mediante la escritura de ideas principales e ideas de apoyo, y de segmentos de superestructura como introducción, desarrollo y conclusiones. Si se trata de un artículo de carácter científico, los estudiantes eventualmente realizan un abstract como modelo general de su escritura.

\subsection{Prueba diagnóstica de lectura.}

La prueba de lectura se diseñó para conocer los desempeños de los estudiantes en cuanto al uso e identificación de los sistemas de representaciones de la lingüística textual y discursiva que fueron considerados en nuestro marco teórico. Se partió del supuesto de que el dominio en la lectura crítica de dichos sistemas de representaciones es correlato (aunque no necesariamente lineal) de su capacidad para ponerlos en uso en el momento de redactar textos aca- 
démicos.

El corpus a ser analizado estuvo integrado por dos artículos de opinión cortos, tomados de publicaciones periódicas colombianas (la revista Semana, y el portal en línea Kienyke): La columna de opinión titulada “¡Ay, los toritos!” de Antonio Caballero, y la columna de opinión titulada “¡Ay, los toritos! ¿Libres?”, de Margarita Londoño. La elección se debió a que ambos textos abordan una misma problemática (la prohibición de las corridas de toros en Santa Fe de Bogotá) desde perspectivas opuestas, lo que permitía sondear en profundidad la apropiación de los mecanismos de la argumentación por parte de los estudiantes, en la medida en que explicitaba las oposiciones que en un solo texto no necesariamente estarían expuestas.

El diagnóstico fue realizado a 48 estudiantes, y entre los resultados se encontró que, en cuanto al texto de Caballero, solo el 10\% de ellos identificó "lo enunciado" (o tema), mientras que, en el texto de Londoño, solo un 23\% realizó dicha identificación. Los resultados contrastan con las afirmaciones de los estudiantes durante la encuesta, en la que el 93\% indicó que identificaba los temas de los textos.

En lo que concierne a la identificación de los enunciadores, un 79\% pudo llevar a cabo esta acción en el texto de Caballero, mientras que solo un 42\% lo hizo en el texto de Londoño. Esto puede deberse a que en el texto de Caballero está expresada de un modo más claro la oposición fundamental en torno a la cual gira el debate.

Por otra parte, solo un $10 \%$ de los estudiantes identificó la proposición/tesis del texto de Antonio Caballero, mientras que un 48\% identificó la proposición/tesis del texto de Londoño. Parece manifestarse así una discrepancia con respecto al conocimiento adquirido en el curso de COE II, que hace especial énfasis en esta noción. Así mismo, la diferencia en los desempeños frente a ambos textos puede confrontarse con los datos de la encuesta que muestran variación en cuanto a la identificación de los distintos elementos que conforman la red argumentativa. Si los estudiantes no tienen claro el modelo y la función de cada uno de sus elementos, el resultado es que su comprensión del sistema no resulta del todo coherente, y se dificulta la transferencia del conocimiento teórico al ejercicio con textos concretos.

Es posible que prime un modelo de apropiación lineal de las categorías de tesis y datos, que son los que los estudiantes dicen reconocer con mayor facilidad. Y es claro que, si se aborda la argumentación en forma simplificada, los desempeños de los estudiantes van a estar por debajo del nivel de experticia al que apunta la alfabetización académica.

No obstante, podría ocurrir que las demandas de escritura que se hacen a los estudiantes a lo largo de sus carreras hagan que no sean requeridos esos niveles de comprensión de las estructuras argumentativas. Es decir, en términos prácticos, los estudiantes serían exitosos con una representación de la argumentación y de su funcionamiento lingüístico, textual y discursivo que, aunque aparece en el lugar del modelo que se enseña, dista mucho del mode- 
lo mismo.

En cuanto a la identificación de puntos de vista opuestos a los de los enunciadores principales, un $60 \%$ pudo hacerlo en el texto de Caballero. Esto puede deberse, como anteriormente se mencionó, al énfasis que se da al aspecto dentro del artículo. En lo que concierne al texto de Londoño, apenas el 50\% identificó el punto de vista opuesto al del enunciador principal. Los datos varían considerablemente cuando se los compara con los que corresponden a las tesis. Dicho fenómeno podría deberse, una vez más, a la falta de comprensión de la estructura lógica de un modelo argumentativo como el que se trabaja en el curso de COE II.

Complementariamente, el 6\% de los estudiantes identificó la proposición-dato en el texto de Caballero y un $10 \%$ lo hizo en el de Londoño. Tal proposición permite inferir el punto de vista opuesto al del enunciador principal en la red argumentativa de los textos. Esto apuntaría una vez a la sospecha de que el modelo aprendido por los estudiantes es lineal, lo que supondría, a su vez, una inadecuación entre sus representaciones sobre lo que es la argumentación y cómo funciona, y las estructuras argumentativas tal y como han sido analizadas por los expertos.

Así mismo, solo un 38\% identificó la proposición-dato del que se deriva el punto de vista del enunciador principal en el texto de Caballero; y sólo un 58\% hizo lo mismo con el texto de Londoño. Se deja ver aquí la falta de comprensión, por parte de los estudiantes, no solo de las estructuras de la argumentación, sino también de la estructura semántica de los textos y, con ello, de la dimensión proposicional de la producción de sentido, que no es lo mismo que la dimensión gramatical y frástica. De hecho, cabe la sospecha de que las lecturas corren curso más cerca del nivel gramatical y frástico, que del nivel proposicional y discursivo, lo que se condice con los hallazgos de los estudios que fueron presentados entre los antecedentes de este trabajo.

Por otra parte, en lo que concierne a la identificación del garante de la estructura argumentativa principal se encontró que un 23\% de los estudiantes realizó acertadamente esta acción con el texto de Caballero, y un 38\% lo hizo con el texto de Londoño. Mientras que, en lo que respecta a la proposición que sirve como respaldo para el garante en ambos textos, el $38 \%$ lo hizo acertadamente en el texto de Caballero, mientras que un 23\% lo hizo en el texto de Londoño.

Tal subconjunto de datos permitiría aventurar la hipótesis de que, efectivamente, los estudiantes están adquiriendo una representación lineal de la estructura argumentativa, simplificada al extremo de considerar relaciones estrictamente limitadas a la diada dato-tesis. Así mismo, dada la naturaleza valorativa e institucional de los respaldos, que no necesariamente se manifiesta en la dimensión frástica de los enunciados, apoyaría la hipótesis de que los estudiantes no se apropian de una lectura proposicional y discursiva durante los cursos de 
COE y que, dado su avance académico, no la necesitan. En su lugar, dependerían de las demandas concretas de escritura que se les hagan dentro de los cursos que toman.

A su vez, a propósito de la identificación de la proposición que funciona como reserva, se encontró que el $60 \%$ de los estudiantes consiguió identificarla en el texto de Caballero, mientras que el 35\% lo hizo en el texto de Londoño. Y, en el caso de la identificación de las voces que son convocadas en el texto, el 75\% lo hizo acertadamente en el texto de Caballero, mientras que un $48 \%$ lo hizo en el texto de Londoño. El relativo aumento del desempeño puede deberse al hecho de que, en las prácticas de los cursos COE la noción de "voces" es trabajada con un especial énfasis; y al hecho de que la identificación de las mismas puede realizarse siguiendo marcadores textuales como las referencias a personas o instituciones a las cuales se les adjudica un discurso referido directo o indirecto.

Por otra parte, cuando a los estudiantes se les pidió identificar el propósito de los textos, el 29\% pudo hacerlo acertadamente con el texto de Londoño y el 27\% con el texto de Caballero. Esto confirmaría vacíos en la apropiación de una perspectiva pragmática y crítica del discurso, la comunicación (especialmente escrita) y la producción de sentido. También puede deberse a aspectos concretos de los contratos didácticos de los cursos de COE y de los cursos restantes o a hábitos y prácticas de lectura y escritura generalizados por parte de los estudiantes, en los cuales prima la representación de los textos ligada más a la de estructuras lingüísticas y gramaticales que a las discursivas.

Un desempeño similar se repite cuando se pide a los estudiantes que identifiquen la caracterización más adecuada del enunciatario construido en el texto de Caballero. En tal caso, solo el 35\% de los estudiantes pudo hacerlo de manera acertada.

\subsection{Prueba diagnóstica de escritura}

Por cuanto la prueba de escritura no fue aplicada en la primera fase, el presente documento no incorpora comentarios al respecto. Tal como tuvimos la oportunidad de indicar al comienzo, nuestro informe constituye un avance de la investigación, que planea realizarse en varias fases durante los siguientes semestres.

\section{Conclusión}

Los cursos de COE parecen adecuarse a las demandas que, en la práctica académica concreta, se hace de la escritura de los estudiantes, lo cual se manifestaría en el hecho de que estos avanzan académicamente y no se quedan estancados a lo largo de sus carreras. No obstante, podría existir una discontinuidad entre el contrato escrito del curso, su orientación teórica, las prácticas de enseñanza y aprendizaje que se llevan a cabo, las prácticas de lectura y escritura que se adecuan a las exigencias de los demás cursos, y las representaciones que tienen estudiantes y profesores de las dinámicas y las estructuras de la escritura y la lectura acadé- 
micas.

Para avanzar en la identificación de los factores internos y externos que inciden en el impacto que tienen estos cursos dentro del contexto académico, se hace necesario, por tanto, profundizar en aspectos propios de los contratos didácticos de diferentes cursos ajenos a COE, con el fin de contrastar los datos así obtenidos con los que aquí se presentan. Así mismo, se hace necesario el diseño de una estrategia de abordaje etnográfico de los sujetos, centrada en sus representaciones y prácticas en torno a la lectura y la escritura en contexto extracadémico, con el fin de buscar la existencia de correlaciones entre hábitos lectores y de escritura, sociabilidades, y desempeños en la academia y en pruebas diagnósticas. Para ello se requiere de una reformulación de la metodología de esta investigación, la cual se llevará a cabo a comienzos del año 2015.

De momento, los datos parecen indicar que existen dos niveles de impacto de los cursos COE. En el primero, los estudiantes, legitimados por la calificación que indica que han conseguido los objetivos del curso, se conciben a sí mismos como sujetos que se han apropiado los rudimentos de la escritura y la lectura académicas. En el segundo, ligado al desempeño en prueba diagnóstica, se manifiesta un panorama diferente caracterizado por mostrar una apropiación limitada de las estructuras de los textos y centrado en la noción de párrafo, no aborda los aspectos macroproposicionales que dan cuenta de la estructura semántica de los dispositivos textuales ni de la progresión temática. Así mismo, se identifica una ruptura entre estructuras simplificadas y lineales de la argumentación que los estudiantes apropian durante los cursos COE (reducidas a la relación inmediata entre dato y tesis), y las estructuras que supuestamente son trabajadas en los mismos tal y como han sido analizadas por los teóricos de la argumentación, y en especial Toulmin.

La no existencia de malestar o de evidencias previas de esta disociación podría estar dando cuenta de una cultura académica en la que la escritura y la lectura son comprendidas, por fuera del discurso explícito e institucional, como prácticas distintas a aquellas que son promulgadas por los discursos y estudios de los expertos en la alfabetización académica. Estaríamos pues, ante una muestra paradigmática de las discontinuidades entre discurso institucional y representaciones de los sujetos, por un lado, y prácticas concretas, por el otro.

Tal discontinuidad, así como los mecanismos que la legitiman y los hábitos que subyacen a ella, constituirían el eje investigativo en torno a lectura y escritura que se espera desarrollar en la Universidad Icesi; y ,con ello, marcar un rumbo alterno en el ámbito nacional, al cada vez más tradicional enfoque que se centra en fines de aprendizaje desde una perspectiva lineal y didáctica de la educación, que no consulta el aporte de las ciencias sociales a la comprensión de las dinámicas que acercan y alejan los discursos y las representaciones de lo que hacen las personas y las instituciones con aquello que constituye su tarea académica, institucional y social. 


\section{Bibliografía}

ADAM, J. M. (1995). Hacia una definición de la secuencia argumentativa. Aprendizaje. Comunicación, lenguaje y educación.

ARIAS A., V., AGUDELO M. C. (2010) ¿La lectura y la escritura de la universidad colombiana corresponde con el proyecto de la modernidad? Latinoam.estud.educ. Manizales (Colombia), 6 (1): 95 - 109, enero - junio de 2010.

BRASLAVSKY, B. (2003) ¿Qué se entiende por alfabetización? Lectura y vida, 24 (2), 6-21.

Carlino, Paula. “QQué nos dicen las investigaciones internacionales sobre escritura en la universidad?" Cuadernos de psicopedagogía, 4, pp. 21-40. Publicación anual de la Escuela de Psicopedagogía de la Universidad Pedagógica y Tecnológica de Colombia (ISSN 1794-9580), 2007. Tunja, Boyacá, Colombia.

AUSTIN, J.L. (1962). "How to do things with words. The William James lectures delivered at Harvard University in 1955". Oxford Clarendon Press, Oxford University Press, Amen House, London, E.C. 4.

CALSAMigliA, H. y TUSÓN, A. (2001) Las cosas del decir. Manual del análisis del discurso. Barcelona: Editorial Ariel.

CARBONÓ-TRUYOL, V. \& MIRANDA-TRUJILLO, R. (2008). La comprensión lectora en los estudiantes de la Universidad del Atlántico: un estudio para perfilar estrategias conducentes a su desarrollo. II Encuentro Nacional y I Internacional sobre Lectura y Escritura en Educación Superior. Bogotá.

CARLINO, Paula. (2005). Escribir, leer y aprender. Una introducción a la alfabetización académica. Buenos Aires: Fondo de Cultura Económica.

CARLINO, Paula. (2006). Escribir, leer y aprender en la universidad. Fondo de cultura económica de Argentina.

CARVAJAL BARRIOS, G. \& ULLOA SANMIGUEL, A. (2009) "La enunciación en la construcción del texto escrito: Formulación conceptual y pertinencia en una investigación sobre cultura escrita, conocimiento y tecnocultura en la Universidad". En: Colombia Nexus ISSN: 19009909 ed: Centro Editorial Universidad Del Valle v.5 fasc. N/A p.26 - 53

(2009). La enunciación en la construcción del texto escrito: formulación conceptual y pertinencia en una investigación sobre cultura escrita y conocimiento en la universidad. En: Revista Nexus, Universidad del Valle, No. 5.

CHARADEAU, Patrick. (2004). La problemática de los géneros: De la situación a la construc- 
ción textual. Revista signos, 37(56), 23-39. Recuperado en 03 de octubre de 2013, de http:// www.scielo.cl/scielo.php?script=sci_arttext\&pid=S0718-

$09342004005600003 \& \operatorname{lng}=$ es\&tlng=es. 10.4067/S0718-09342004005600003.

GONZÁLEZ, Hipólito (2008). Cartilla docente. El proyecto educativo de la Universidad Icesi y el aprendizaje activo. Centro de recursos para la enseñanza y el aprendizaje. Universidad Icesi, Cali.

GRAPHOS (2008). Problematizar la lectura y escritura académicas en la universidad. II Encuentro Nacional y I Internacional sobre Lectura y Escritura en Educación Superior. Bogotá.

LITWIN, E. (1997). Las configuraciones didácticas. Una nueva agenda para la enseñanza superior. Buenos Aires: Paidós.

MARÍN, M. y HALL. B (2005). Prácticas de lectura con textos de estudio. Buenos Aires: Eudeba.

MARÍN, Martha, (2006). Alfabetización académica temprana. Argentina: Lectura y vida.

RINCON BONILLA, G., GIL ROJAS, J., S. (2010). "Las prácticas de lectura y de escritura académicas en la Universidad del Valle: tendencias". Lenguaje, 38 (2).

MARTíNEZ M. C. (2002). Lectura y escritura de textos. Perspectivas teóricas y talleres. Cali: Cátedra UNESCO para la lectura y la escritura. Universidad del Valle.

MARTínEZ, M.C. (2005). La construcción del Proceso Argumentativo en el Discurso. Perspectivas Teóricas y Trabajos Prácticos. Cali: Cátedra Unesco, Universidad del Valle.

NAVARRETE, P. (2008). Programa LEA (lectoescritura y aprendizaje en la Universidad de Santander). II Encuentro Nacional y I Internacional sobre Lectura y Escritura en Educación Superior. Bogotá.

OVIEDO Tito Nelson (1997); Hacia Una Base Semántico Comunicativa para la Gramática. Universidad del Valle. Cali

OViEDO, Tito Nelson (2003) Abra la boca... significación comunicación. En Revista Lenguaje No. 31. Universidad del Valle. Cali

OVIEDO, Tito (2012). Evaluación de trabajos escritos en los cursos de comunicación oral y escrita. Departamento de Español. Universidad Icesi, Cali.

PÉREZ-ABRIL, M. \& RINCÓN-BONILLA, G. (Coords.) (2013) ¿Para qué se lee y se escribe en la universidad colombiana? Un aporte a la consolidación de la cultura académica del país. Bogotá: Editorial Pontificia Universidad Javeriana. 
SAN MARTÍN, J. (2009). UBA - Facultad de Ciencias Sociales - Informe final de investigación - Noviembre de 2009 "Escribir, leer y aprender en la Universidad" Una introducción a la alfabetización académica de la Dra. Paula Carlino.

SEARLE, John R. (1999). "Expression and meaning. Estudies in the theory of speech acts". Cambridge University Press, Cambridge.

SEARLE, John R. (1962). “QQué es un acto de habla?”. En: VALDÉS, Villanueva, Luis ML (1995). "La búsqueda del significado. Lecturas de filosofía del lenguaje". Editorial Tecnos, Madrid.

URIBE-ÁLVAREZ, G. \& CAMARGO-MARTÍNEZ, Z. (2008). Prácticas de lectura y escritura académicas en la universidad colombiana. magis, Revista Internacional de Investigación en Educación, 3 (6), 317-341.

\section{Grupo de Investigación en Recursos y Teorías para el Aprendizaje (IRTA)}

https://bit.ly/2NPBvrn

IRTA es el grupo de investigación de la Escuela de Ciencias de la Educación de la Universidad Icesi. Tiene como objetivos construir propuestas teórico metodológicas, para diversos campos del saber, que los docentes de las diferentes disciplinas puedan utilizar para alcanzar los objetivos de formación y aprendizaje propuestos en sus procesos de enseñanza y aprendizaje; definir modelos para validar y verificar la operacionalización de los conceptos y fundamentos del aprendizaje contenidos en el proyecto educativo de la Universidad; y, estructurar un conjunto de métodos, técnicas y actividades para guiar la aplicación de las tecnologías de información y comunicación (TIC) en procesos de aprendizaje. Desarrolla investigaciones en los campos educativo y pedagógico, así como investigaciones aplicadas e intervenciones que buscan incidir y transformar las realidades educativas.

\section{LIINEAS DE INVESTIGACIÓN}

1. Didácticas, pedagogía y aprendizajes

2. Currículo y evaluación

3. TIC y educación

4. Entornos, gestión y políticas educativas

5. Experiencias de formación y transformación educativa: sujetos y

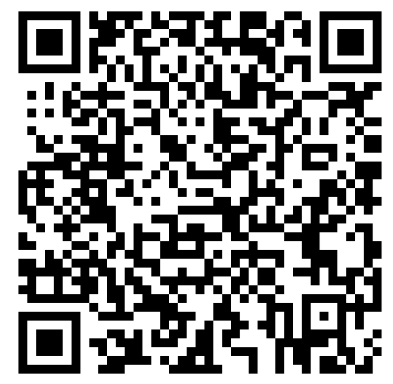


